\title{
Discovery of calcite in the solar type protostar NGC 1333-IRAS 4
}

\author{
C. Ceccarelli ${ }^{1,2}$, E. Caux ${ }^{3}$, A. G. G. M. Tielens ${ }^{4}$, F. Kemper ${ }^{5}$, L. B. F. M. Waters ${ }^{6}$, and T. Phillips ${ }^{7}$ \\ 1 Observatoire de Bordeaux, BP 89, 33270 Floirac, France \\ 2 Laboratoire d'Astrophysique de l'Observatoire de Grenoble, France \\ 3 CESR CNRS-UPS, BP 4346, 31028 Toulouse Cedex 04, France \\ ${ }^{4}$ SRON, PO Box 800, 9700 AV Groningen, The Netherlands \\ 5 Astronomical Institute Anton Pannekoek, Univeristy of Amsterdam, Kruislaan 403, 1098 SJ Amsterdam, The Netherlands \\ 6 Instituut voor Sterrenkunde, KU Leuven, Celestijnenlaan 200B, 3001 Heverlee, Belgium \\ 7 California Institute of Technology, Downs Laboratory of Physics 320-47, Pasadena, CA 91125, USA
}

Received 26 August 2002 / Accepted 11 October 2002

\begin{abstract}
We present observations, obtained with ISO-LWS, of the continuum between 50-200 $\mu \mathrm{m}$ of the solar type protostar IRAS 4, in the NGC 1333 complex. The continuum presents an excess, around $95 \mu \mathrm{m}$, that we demonstrate must be a dust feature. We compared the $95 \mu \mathrm{m}$ excess with the calcite feature at $92 \mu \mathrm{m}$ and find that it fits the observations reasonably well. There may be a further contribution from hydrous silicates at $\sim 100 \mu \mathrm{m}$, but this seems a less robust result. The detected calcite mass is $\sim 8 \times 10^{-5} M_{\odot}$ and represents about $1 \%$ of the warm $(\sim 23 \mathrm{~K})$ dust mass surrounding IRAS 4 . This is only the second observation indicating the presence of carbonates outside the solar system, and the first revealing calcite in a young protostar. It is remarkable and intriguing that in all the objects where calcite has been detected so far, namely meteorites, planetary nebulae and IRAS 4, it represents from 0.3 to $1 \%$ of the dust mass. This new detection of calcite strengthens the claim by Kemper et al. (2002a) that calcite formation does not necessarely requires liquid water. We suggest that calcite forms at the surface of the grains, where water ice layers may locally have an enhanced mobility caused by heating due to hard X-rays emitted by the central object.
\end{abstract}

Key words. ISM: abundances - ISM: molecules - stars: formation - ISM: individual: IRAS 4

\section{Introduction}

Carbonates are a common mineral in Solar system bodies such as meteorites and interplanetary dust particles (IDPs). In particular, CI carbonaceous meteorites show a complex set of fractures filled with carbonates, hydrous calcium, and magnesium sulfates. These meteorites consist otherwise largely of layer lattice silicates. The petrographic structure of these meteoritic carbonates reveals an origin in hydrothermal alteration followed by extensive brecciation on the parent body presumably at the time of its formation (Richardson 1978; Macdougall et al. 1984). The origin of the carbonate phases in the IDPs is less clear. Given the dominance of hydrated silicates in the IDPs with carbonates, this class of IDPs probably derives from asteroidal parent bodies rather than comets (Sandford 1986). Carbonates have also been discovered in martian meteorites (Gooding et al. 1988; Gooding et al. 1991; Treiman et al. 1993) and interpreted as one of the pieces of evidence for liquid water (Wentworth \& Gooding 1994; Bridges et al. 2001) and presence of life on Mars (McKay et al. 1996). That interpretation is

Send offprint requests to: $\mathrm{C}$. Ceccarelli, e-mail: ceccarel@observ.u-bordeaux.fr however very controversial (e.g. Steele et al. 2000; Farquhar \& Thiemens 2000).

Apart from the detection by in situ analysis, as is the case of the carbonates in meteorites, solid material from outside the solar system can only be revealed by the spectroscopic observation of characteristic bands, either in emission or absorption. Recent laboratory spectra of carbonates show bands in the far infrared $(\sim 30-100 \mu \mathrm{m})$ for some common carbonates: calcite, and dolomite (Kemper et al. 2002a; hereinafter KJW2002: see also Kemper et al. 2002b). This wavelength range is totally obscured by the terrestrial atmosphere, and only recently spectroscopic observations in this range have been possible with the two spectrometers on board the Infrared Space Observatory (ISO; Kessler et al. 1996). As a consequence, the first detection of carbonates outside the solar system, and specifically in two planetary nebulae by KJW2002, has just happened this year. This discovery represents a big challenge to theories of carbonate formation, as obviously no liquid water is present in such objects.

In this Letter we present evidence for carbonates in a low mass protostar, NGC 1333-IRAS 4, which represents a third specific category of objects, having physical conditions 
drastically different from both Solar System objects and the two planetary nebulae. IRAS 4 is in fact a very embedded protostar, surrounded by a massive envelope in the NGC 1333 cloud, belonging to the Perseus complex (Jennings et al. 1987). IRAS 4 is among the coldest and probably youngest protostars so far known. It is a binary system (IRAS 4 A and B; Sandell et al. 1991 ) with a total bolometric luminosity of $11 L_{\odot}$ (distance of $220 \mathrm{pc}$; Cernis 1990), widely studied in the millimeter wavelengths (e.g. Blake et al. 1995; Lefloch et al. 1998). Its envelope and surrounding is relatively rich in water vapor (Ceccarelli et al. 1999; Maret et al. 2002; Bergin et al. 2002).

\section{Observations and data reduction}

The observations were performed using the Long Wavelength Spectrometer instrument (hereafter LWS: Clegg et al. 1996) on the ISO satellite. These observations, obtained during revolution 847 in the grating mode (LW01), were performed for three positions. One centered on the coordinates: $\alpha_{2000}=$ $03^{\mathrm{h}} 29^{\mathrm{m}} 11.9^{\mathrm{s}}, \delta_{2000}=31^{\circ} 13^{\prime} 20.3^{\prime \prime}$, in between IRAS $4 \mathrm{~A}$ and IRAS 4B, and hence the LWS beam $\left(80^{\prime \prime}\right)$ includes both sources. The other two positions targeted the red-shifted and blue-shifted lobe peaks of the outflow powered by IRAS 4: NE-red $\left(\alpha_{2000}=03^{\mathrm{h}} 29^{\mathrm{m}} 15.6^{\mathrm{s}}, \delta_{2000}=31^{\circ} 14^{\prime} 40.1^{\prime \prime}\right)$ and SW-blue $\left(\alpha_{2000}=03^{\mathrm{h}} 29^{\mathrm{m}} 06.6^{\mathrm{s}}, \delta_{2000}=31^{\circ} 12^{\prime} 08.7^{\prime \prime}\right)$. At all three positions, the full LWS wavelength coverage was used (43-196 $\mu \mathrm{m}$ ), with a resolving power of about 200 . The central position observation is made up of 30 scans while both "off" position observations consist of 10 scans, all of them sampled at $1 / 4$ of the grating resolution $(0.075 \mu \mathrm{m}$ in the $43-$ $92 \mu \mathrm{m}$ range and $0.15 \mu \mathrm{m}$ in the $92-196 \mu \mathrm{m}$ range; Gry et al. 2001), yielding integration times per spectral element of $48 \mathrm{~s}$ for the central position and $16 \mathrm{~s}$ for the two off source positions. The data processing was carried out using a new routine of the LWS Interactive Analysis (LIA) software, allowing the correction of the detector's transient effects (Caux 2001). However, we emphasize that the derived continuum spectrum is substantially identical to that obtained when the standard ISOLWS Off-Line Processing software (OLP v10) is used. The final analysis was made using the ISAP package, to remove bad data points at the edges of each detector, and to perform a "stitching" of the individual detector spectra. ISO LW01 spectra are flux calibrated using Uranus, and the final accuracy on the absolute flux is estimated to be better than $30 \%$ (Swinyard et al. 1998).

As will be discussed in the next section, we have detected a dust feature between 90 and $100 \mu \mathrm{m}$. We are confident that the observed feature is real, and is not the result of instrumental effects, for the following reasons. First, it is present whatever the version of the OLP used. Second, spurious features have been observed in some LWS grating spectra, but only on sources much brighter than NGC 1333 IRAS 4. Finally, to remove suspicion that the feature is an artificial result of our stitching method, note that the $90-100 \mu \mathrm{m}$ range is entirely observed by one LWS detector, i.e. the detector LW5 which covers the range $83-110 \mu \mathrm{m}$. The two adjacent detectors cover, however, most of the LW5 wavelength range: SW1 covers the 78-93 $\mu \mathrm{m}$ range, and LW4 the 103-130 $\mu \mathrm{m}$ range respectively, and themselves

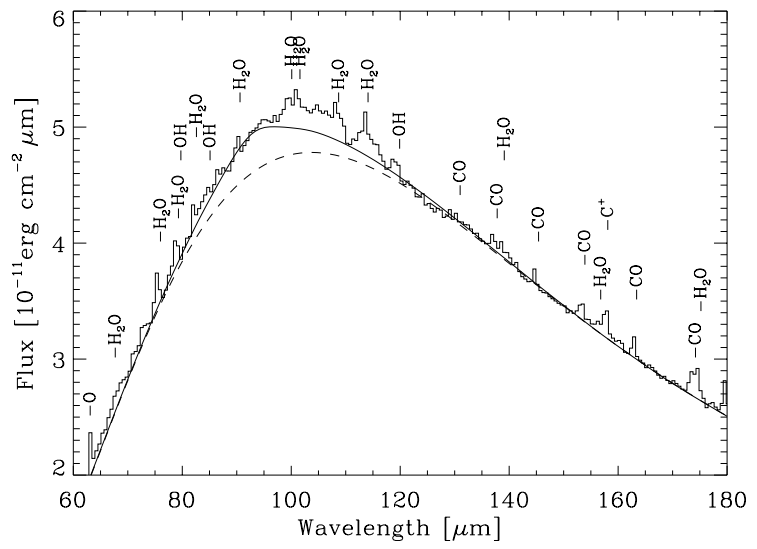

Fig. 1. The ISO-LWS observed spectrum of IRAS 4 (solid line). The dashed line shows the best fit obtained with a two (three) grey bodies procedure (see text). The thin solid line shows the best fit obtained adding the calcite (see text). Several lines are visible and are marked according to the chemical species $\left(\mathrm{O}, \mathrm{C}^{+}, \mathrm{H}_{2} \mathrm{O}, \mathrm{CO}\right.$ and $\mathrm{OH}$ respectively).

are largely overlapped by adjacent detectors too. It is therefore virtually impossible that the feature is artificial, caused by our stitching method.

\section{Data analysis}

The resulting spectrum of IRAS 4 is shown in Fig. 1. Superimposed with the dust continuum many lines are visible, namely $\mathrm{H}_{2} \mathrm{O}, \mathrm{CO}, \mathrm{OH}, \mathrm{O}$ and $\mathrm{C}^{+}$lines. Here we focus on the continuum and refer the interested reader to Maret et al. (2002) for a detailed discussion of the line spectrum.

Young protostars of the type of IRAS 4 are heavily embedded in their placental envelopes, which completely obscure, optically, the new born interior object (they are usually referred as Class 0 sources; e.g. André et al. 2000). Their FIR $(\lambda \geq 60 \mu \mathrm{m})$ to millimeter continuum spectrum is often well described by a single blackbody multiplied by the dust opacity $k_{\lambda}$, which in these wavelength range is typically approximated by a power law $k_{\lambda}=k_{0}\left(\lambda_{0} / \lambda\right)^{\beta}$ (e.g. Henning et al. 1995). The value of $\beta$ depends on the dust composition, shape and size (coagulation and mantle formation), and varies between 1 to 2 in Class 0 sources (e.g. Chandler \& Richer 2000; see also the theoretical study by Ossenkopf \& Henning 1994). In the following we will refer to this as a "greybody", for brevity, although strictly speaking a greybody has a constant wavelength-independent opacity, whereas we use a power law dust opacity here. Rarely is more than one greybody necessary to reproduce the FIR spectrum of Class 0 sources (e.g. André et al. 2000). In the specific case of IRAS 4 the continuum emission is probably contaminated by the cold emission of its parental cloud at wavelengths longer than $140 \mu \mathrm{m}$. We therefore modeled the IRAS 4 continuum emission as due to two greybodies where we minimized the $\chi^{2}$, as usually defined as the sum of the squares of the difference of the theoretical and observed values, normalized to the number of points. 
Table 1. Parameters of the best fit theoretical emission, obtained by adding up two greybodies (first two columns) and three greybodies (last three columns) respectively.

\begin{tabular}{c|rr|rrr}
\hline \hline & 1 & 2 & 1 & 2 & 3 \\
\hline$\tau(60 \mu \mathrm{m})$ & 0.30 & 0.35 & 0.30 & 0.42 & 0.35 \\
$\beta$ & 1.0 & 1.2 & 1.0 & 1.5 & 1.2 \\
$T(\mathrm{~K})$ & 10 & 23 & 10 & 18 & 23 \\
$\theta\left({ }^{\prime \prime}\right)$ & 80 & 23 & 80 & 4 & 23 \\
Dust mass $\left(0.01 M_{\odot}\right)$ & 7.8 & 0.75 & 7.8 & 0.03 & 0.75 \\
\hline
\end{tabular}

In practice we used the following expression for computing the theoretical continuum emission $I_{\lambda}$ :

$I_{\lambda}=\sum_{i} B B\left(\lambda, T_{i}\right) \cdot\left(1-\exp \left[-\tau_{i}(60 \mu \mathrm{m}) \cdot\left(60 / \lambda_{\mu \mathrm{m}}\right)^{\beta_{i}}\right]\right) \cdot \Omega_{i}$

with the sum running from 1 to 2 , and where $B B\left(\lambda, T_{i}\right)$ is the blackbody at temperature $T_{i}$ and wavelength $\lambda$. The free parameters are the opacities $\tau_{i}$ of the two greybodies, the power law coefficients $\beta_{i}$, the temperatures $T_{i}$, and the solid angles $\Omega_{i} \sim \theta_{i}^{2}$. The best fit is obtained with the parameters in Table 1 and it is shown in Fig. 1. The observed continuum emission is well reproduced by adding up an extended cold component $(10 \mathrm{~K})$ filling up the ISO-LWS beam and a warm ( $23 \mathrm{~K})$, more compact (23" in size) component. The cold component includes both the coldest region of the envelope and the cloud material itself, whereas the warm component is associated with the warm envelope of the protostar. The warm dust mass is $7.5 \times 10^{-3} M_{\odot}$, obtained assuming a mass absorption coefficient of $4 \mathrm{~g}^{-1} \mathrm{~cm}^{2}$ at $60 \mu \mathrm{m}$ (Ossenkopf \& Henning 1994). The uncertainty in the mass absorption coefficient is about a factor two: the value $4 \mathrm{~g}^{-1} \mathrm{~cm}^{2}$ is the average between the values obtained by Ossenkopf \& Henning (1994) for grains with thin and thick mantles respectively, and for a medium density of $10^{6} \mathrm{~cm}^{-3}$, appropriate for the IRAS 4 conditions. Jøgersen et al. (2002) and Maret et al. (2002) modeled the physical structure of IRAS 4 and found substantially the same values that we find for the temperature, extent and mass of the material forming its envelope. Specifically, Jøgersen et al. (2002) found that the dust mass at $10 \mathrm{~K}$ of the IRAS 4 two components amounts to $4.3 \times 10^{-2} M_{\odot}$ (compared to our estimate of $7.8 \times 10^{-2} M_{\odot}$, which, however, includes a larger region). Maret et al. (2002) found a gas mass at $25 \mathrm{~K}$ of about $0.8 M_{\odot}$. Assuming a dust-togas ratio of 1:100 the estimate of the warm dust mass by Maret et al. (2002) agrees extremely well with the present work estimate, i.e. $7.5 \times 10^{-3} M_{\odot}$. Therefore we conclude that the procedure we used to model the continuum gives basically correct results.

The two greybodies reproduce, reasonably well, the observed continuum, except in the 90-110 $\mu \mathrm{m}$ range, which seems to be underestimated. The best fit shown in Fig. 1 has been obtained excluding this region, to find the best fit to the continuum in the remaining wavelengths. Including the 90 $110 \mu \mathrm{m}$ range results in a much worse fit to the continuum at the other wavelengths, without improving the fit in this range. We tried adding a third component, i.e. a third greybody, again leaving the opacities, etc. as free parameters (Table 1). However, this does not change the result appreciably, leaving the excess in the $90-110 \mu \mathrm{m}$ wavelength range substantially the same and unaccounted for. Furthermore, we find that the third component, at $18 \mathrm{~K}$, would originate from a smaller region than that emitting at $23 \mathrm{~K}$, which is not physical. The bottom line is that the excess in the 90-110 $\mu \mathrm{m}$ wavelength range cannot be accounted for by adding a new greybody, or a sum of greybodies. We also applied our procedure to the LWS continuum spectrum of another solar type protostar, IRAS 16293-2422 (data taken from Ceccarelli et al. 1998), which is a Class 0 source, in other aspects similar to IRAS 4 (see e.g. the discussion in Maret et al. 2002). Contrary to IRAS 4, the spectrum of IRAS $16293-2422$ is well fitted by the three greybodies procedure (8, 16 and $28 \mathrm{~K}$ respectively) and absolutely no excess remains in the $90-100 \mu \mathrm{m}$ wavelength range. We also checked the spectra of the two off positions of IRAS 4. Again, we could fit both spectra with our three-greybodies procedure and no residuals show up in the $90-100 \mu \mathrm{m}$ range. We conclude that the 90-100 $\mu \mathrm{m}$ excess observed towards IRAS 4 cannot therefore be an instrumental or model problem and must be a real dust feature.

\section{Calcite}

The question arises as to the carrier of this feature. Not many solids are known to possess bands in the 90-100 $\mu \mathrm{m}$ range. As mentioned in the Introduction, KJW2002 and Kemper et al. (2002b) presented the laboratory spectrum of several carbonates which have spectral features at $\lambda \geq 50 \mu \mathrm{m}$. Also hydrous silicates are known to possess some features longwards $60 \mu \mathrm{m}$ (Koike \& Shibai 1990). Another solid having bands at long wavelengths is $\mathrm{CO}_{2}$ but its two bands (at $114 \mathrm{~cm}^{-1}$ and $68 \mathrm{~cm}^{-1}$; Ron \& Schnepp 1967) fall well away from the observed excess. Of the carbonates reported by KJW2002, calcite ${ }^{1}$ and aragonite are the only ones having a band around $90 \mu \mathrm{m}$. Since the $90 \mu \mathrm{m}$ resonance band of aragonite is much weaker than that of calcite and, furthermore, aragonite can only be formed at high pressures and temperatures, it is unlikely at the origin of the observed feature in IRAS 4. Among the hydrous silicates studied by Koike \& Shibai (1990), montmorillonite is the only one having a resonance in the 90$100 \mu \mathrm{m}$ range, but it is about 20 times weaker than the resonance of the calcite in the same wavelength range.

Figure 2 shows the $90-100 \mu \mathrm{m}$ excess in IRAS 4 and in the planetary nebula NGC 6302 observed by KJW2002, together with the laboratory spectra of calcite and montmorillonite. The similarity of the spectra of IRAS 4 and NGC 6302 is striking. Since KJW2002 successfully fitted the NGC 6302 excess as due to calcite, we tried to fit the IRAS 4 spectrum with the addition of calcite emitting at $23 \mathrm{~K}$. The result is shown in Fig. 1, as a thin solid line. The fit improves substantially: the calcite laboratory spectrum reproduces reasonably well the observed 90-100 $\mu \mathrm{m}$ excess. There may be a further residual longwards of $100 \mu \mathrm{m}$, but given the presence of several strong lines in that range the residual may be mostly caused by those lines.

\footnotetext{
1 Calcite has a second, less intense emission feature around $45 \mu \mathrm{m}$, but unfortunately the continuum of IRAS 4 is by far too weak to verify its existence.
} 


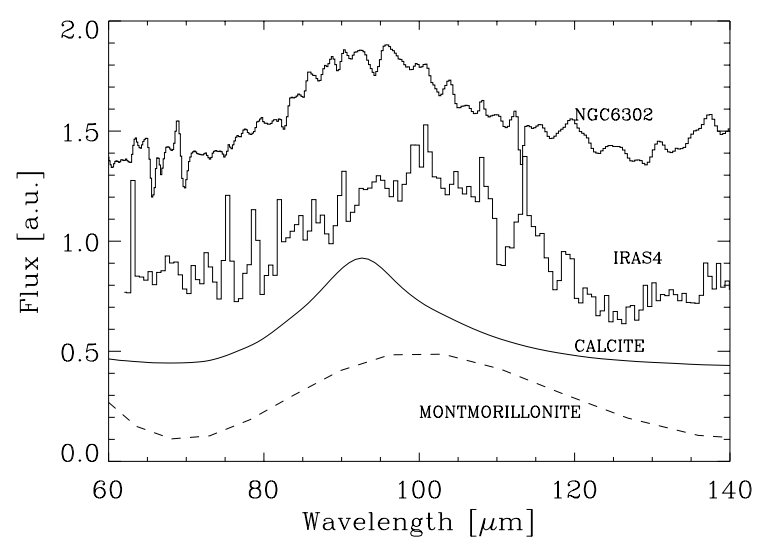

Fig. 2. The 90-100 $\mu$ m excess, after subtraction of the other known dust features and continuum, of the planetary nebula NGC 6302 (top) and the protostar IRAS 4 (middle), compared with the laboratory spectra of calcite and montmorillonite (bottom).

Alternatively, montmorillonite may be responsible for this further excess, but given the presence of so many lines in that range it is impossible to assess the reliability of this suggestion. We wish to emphasize, however, that montmorillonite alone would give a much worse fit than that obtained with calcite, mainly because the montmorillonite feature is too broad with respect to the observed IRAS 4 excess. Although we cannot exclude that the observed feature is indeed due to a different carrier, calcite with perhaps some montmorillonite (in a quantity which could be up about 5 times the calcite) seems at present the best explanation we have for it.

In order to reproduce the observed feature about $8.4 \times$ $10^{-5} M_{\odot}$ (i.e. $27 M_{\oplus}$ ) of calcite, emitting at $23 \mathrm{~K}$, are needed. This is about $1 \%$ of the warm dust mass and $0.01 \%$ of the total dust (cold+warm) of IRAS 4 (with a factor two of uncertainty). Assuming solar abundances $\left(\mathrm{Ca} / \mathrm{H}=2 \times 10^{-6}\right.$; Snow 1984) and a dust to gas ratio equal to $1: 100$, about $40 \%$ of $\mathrm{Ca}$ is locked up in the calcite emitting at $23 \mathrm{~K}$. For comparison, KJW2002 found that about $30 \%$ of $\mathrm{Ca}$ is locked in the calcium-bearing minerals of NGC 6302 (calcite, dolomite and diopside) and that calcite represents about $0.3 \%$ of the dust mass (see also Kemper et al. 2002b). The similarity of these values, within a factor 3, is remarkable and puzzling at the same time, as IRAS 4 and NGC 6302 are such different sources, with very different physical conditions and histories. In carbonaceous meteorites, calcite represents about $1 \%$ (Treiman 1995), which is also the same order of magnitude that we observe in IRAS 4. Finally, it is worth to note that on the Earth, where carbonates form efficiently, the ratio between carbonates and silicates is around $1-2 \%$. Whether this similarity is just a coincidence, or hints to a common formation mechanism, is impossible to say at this stage.

\section{Discussion and conclusions}

As discussed in the Introduction, carbonates in meteorites have been attributed to aqueous alteration of rocky material. On the other hand, liquid water being absent in planetary nebulae, it is very unlikely that the carbonates detected by KJW2002 formed via the same route. KJW2002 suggested three alternatives, of which they considered the most likely to be that the planetary nebulae carbonates formed at the time of the silicate condensation, resulting from the co-condensation of gaseous $\mathrm{CO}_{2}$ and $\mathrm{CaO}$ reacting at high temperatures, $\sim 1000-1200 \mathrm{~K}$ (Pope et al. 1996), and subsequently cooled down. Neither of the two other explanations (aqueous alteration and direct condensation) would work in the IRAS 4 case, as no liquid water is expected to be present nor such high abundances of hot gaseous $\mathrm{CO}_{2}$ and $\mathrm{CaO}$ either. Here we suggest that calcite forms through processing of $\mathrm{CO}_{2}$ and $\mathrm{H}_{2} \mathrm{O}$ containing ices. Possibly, calcite forms through catalytic reactions on hydrated silicate surfaces (Lancet \& Andersen 1970). Alternatively, calcite may form on the grain surfaces (following another route considered and discarded by KJW2002), where the layers of the ice mantles directly adjacent to the grain surface could act as liquid water. Water ices enriched in $\mathrm{CO}_{2}$ would react with the $\mathrm{Ca}^{2+}$ contained in the silicate lattice to form calcite. Both explanations are a priori plausible in the IRAS 4 case, as abundant (from 10 to $40 \%$ of water ice) solid $\mathrm{CO}_{2}$ is usually observed towards young protostars (e.g. de Graauw et al. 1996; Gerakines et al. 1999; Nummelin et al. 2001). Note that, given the relatively weak strengths of the $\mathrm{CO}_{2}$ bands at 68 and $114 \mathrm{~cm}^{-1}$ $\left(1.2 \times 10^{-19}\right.$ and $3 \times 10^{-19} \mathrm{~cm}$ per molecule respectively, i.e. about 250 times weaker than the 4.6 band strength; Ron \& Schnepp 1967) the lack of $\mathrm{CO}_{2}$ features in the IRAS 4 spectrum puts a relatively poor constraint on the $\mathrm{CO}_{2}$ ice column density: $\leq 2 \times 10^{19} \mathrm{~cm}^{-2}$, which translates ${ }^{2}$ into $\mathrm{CO}_{2}$-ice $/ \mathrm{H}_{2} \mathrm{O}-$ ice $\leq$ 0.15 . This value has to be taken with caution though, as the line strengths are uncertain by a factor three and it represents an average along the line of sight.

Although most of the IRAS 4 dust is far colder than $190 \mathrm{~K}$ (below that where the water ice mobility is expected to be virtually zero; Rietmeijer 1985), hard X-rays from the center (protostars are notorious powerful X-rays sources; e.g. Feigelson \& Montmerle 1999) may provide a local heating at the surface of the grain, making the ice layers mobile. Once a hard $\mathrm{X}$-ray hits a grain it can warm up the grain locally (Léger et al. 1986; Najita et al. 2001). For example, Najita et al. (2001) estimated that grains $50 \AA$ in sizes, are heated to more than $120 \mathrm{~K}$ by absorption of photons with energies $\geq 300 \mathrm{eV}$. If the grains are larger, only small regions of the grain will be spot heated. Again Najita et al. estimated that sub-units of the grains $50 \AA$ in size would be spot heated to more than $150 \mathrm{~K}$ with a deposit of $300 \mathrm{eV}$ of energy. Smaller sub-units may reach even larger temperature, depending on the grain structure. However, all those numbers depend on a large number of poorly known parameters (e.g. as the structure of the grains) and it is difficult and beyond the scope of this Letter to quantify this effect better, but we think that grain surface formation is a possible explanation for the calcite around IRAS 4. The same explanation would apply to the formation of the hydrous silicates that we may have detected as well. And finally, this opens up the possibility that pre-biotic or even biotic molecules, which also

2 Taking the IRAS $4 \mathrm{H}_{2}$ column density quoted by Jøgersen et al. (2002), $2.5 \times 10^{24} \mathrm{~cm}^{-2}$ and assuming a typical $\mathrm{H}_{2} \mathrm{O}$ ice abundance containing $10 \%$ of the elemental oxygen, gives an upper limit $\mathrm{CO}_{2}$ ice $/ \mathrm{H}_{2} \mathrm{O}$-ice $\leq 0.15$. 
need aqueous solutions to form, are synthesized on the grain surfaces.

It is not clear at this stage whether IRAS 4 represents a peculiar case with respect to other embedded low mass protostars. A systematic search using the ISO archive is in progress to answer this question. The result may also help to understand the calcite formation mechanism. Meantime, the lack of calcite detection in other protostars with strong mid-IR continua (namely protostars more massive and/or more evolved than IRAS 4) in the $7 \mu \mathrm{m}$ calcite band (which is three times stronger than the $92 \mu \mathrm{m}$ feature), already tell us that there is not much warm calcite around them. Whether this has anything to do with the formation mechanism or dust evolution is at present an open question.

Acknowledgements. F. Kemper and L.B.F.M. Waters acknowledge financial support from an NWO "Pionier" grant.

\section{References}

André, P., Ward-Thompson, D. \& Barsony, M. 2000, Protostars \& Planets IV, ed. V. Mannings, A. P. Boss, \& S. S. Russell, 59

Bergin, E. A., Kaufman, M. J., Melnick, G. J., Snell, R. L., \& Howe, J. E. 2002, ApJ, in press

Blake, G. A., Sandell, G., van Dishoeck, E. F., et al. 1995, ApJ, 441, 689

Bridges, J. C., Catling, D. C., Saxton, J. M., et al. 2001, Space Sci. Rev., 96, 365

Caux, E. 2001, in The Calibration Legacy of the ISO mission, ESA Special Publications Series, Vol. 481

Ceccarelli, C., Caux, E., White, G. J., et al. 1998, A\&A, 331, 372

Ceccarelli, C., Caux, E., Loinard, L., et al. 1999, A\&A, 342, L21

Cernis, K. 1990, Ap\&SS, 166, 315

Chandler, C. J., \& Richer, J. S. 2000, ApJ, 530, 851

Clegg, P. E. C., et al. 1996, A\&A, 315, L38

de Graauw, T., et al. 1996, A\&A, 315, L345

Farquhar, J., \& Thiemens, M. H. 2000, J. Geophys. Res., 105, 11991

Feigelson, E. D., \& Montmerle, T. 1999, ARA\&A, 37, 363

Gerakines, P. A., Whittet, D. C. B., Ehrenfreund, P., et al. 1999, ApJ, 522,357

Gooding, J. L., Wentworth, S. J., \& Zolensky, M. E. 1988, Geochim. Cosmochim. Acta, 52, 909

Gooding, J. L., Wentworth, S. J., \& Zolensky, M. E. 1991, Meteoritics, 26,135
Gry, C., Swinyard, B. M., Andrew, A., et al. 2001, ESA Publ. SAI-99-077

Henning, T., Begemann, B., Mutschke, H., \& Dorschner, J. 1995, A\&AS, 112, 143

Jennings, R. E., Cameron, D. H. M., Cudlip, W., \& Hirst, C. 1987, MNRAS, 226, 461

Jøgersen, K., Schöier, F. L., \& van Dishoeck, E. F. 2002, A\&A, 389, 908

Kemper, F., Jager, C., Waters, L. B. F. M., et al. 2002, Nature, 415, 295 (KJW2002)

Kemper, F., Molster, F. J., Jager, C., \& Waters, L. B. F. M. 2002b, A\&A, in press

Kessler, M. F., Steinz, J. A., Anderegg, M. E., et al. 1996, A\&A, 315, L27

Koike, C., \& Shibai, H. 1990, MNRAS, 246, 332

Lancet, M. S., \& Anders, E. 1970, Science, 170, 980

Léger, A., Jura, M., \& Omont, A. 1985, A\&A, 144, 147

Lefloch, B., Castets, A., Cernicharo, J., Langer, W. D., \& Zylka, R. 1998, A\&A, 334, 269

Macdougall, J. D., Lugmair, G. W., \& Kerridge, J. F. 1984, Nature, 307, 249

Maret, S., Ceccarelli, C., Caux, E., Tielens, A. G. G. M., \& Castets, A. 2002, A\&A, 395, 573

McKay, D. S., Gibson, E. K., Thomas-Keptra, K. L., et al. 1996, Science, 273, 924

Najita, J., Bergin, E. A., \& Ullom, J. N. 2001, ApJ, 561, 880

Nummelin, A., Whittet, D. C. B., Gibb, E. L., Gerakines, P. A., \& Chiar, J. E. 2001, ApJ, 558, 185

Ossenkopf, V., \& Henning, T. 1994, A\&A, 291, 943

Pope, K. O., Ocampo, A. C., Fischer, A. G., Morrison, J., \& Sharp, Z. 1996, Lunar Planet. Sci., 27, 1045

Richardson, S. M. 1978, Meteoritics, 13, 141

Rietmeijer, F. J. M. 1985, Nature, 313, 293

Ron, A., \& Schnepp, O. 1967, JCP, 46, 3991

Sandell, G., Aspin, C., Duncan, W. D., Russell, A. P. G., \& Robson, E. I. 1991, ApJ, 376, L17

Sandford, S. A. 1986, Science, 231, 1540

Snow, T. P. 1984, ApJ, 287, 238

Steele, A., Goddard, D. T., Stapleton, D., et al. 2000, Met. planet. Sci., 35,237

Swinyard, B. M., Burgdorf, M. J., Clegg, P. E., et al. 1998, SPIE, 3354, 888

Treiman, A. H., Barrett, R. A., \& Gooding, J. L. 1993, Metoritics, 28, 86

Treiman, A. H. 1995, Metoritics, 30, 294

Wentworth, S. J., \& Gooding, J. L. 1994, Metoritics, 29, 861 